\title{
(6) OPEN ACCESS Temporal ocular coherence tomography-measured
changes in anterior chamber angle and diurnal
intraocular pressure after laser iridoplasty: IMPACT
study
}

\author{
Rupert R A Bourne, ${ }^{1,2}$ Ivailo Zhekov, ${ }^{1,2}$ Shahina Pardhan ${ }^{1}$
}

\begin{abstract}
- Additional material is published online only. To view please visit the journal online (http://dx.doi.org/10.1136/ bjophthalmol-2016-308720).
\end{abstract}

'Vision \& Eye Research Unit, Postgraduate Medical Institute, Anglia Ruskin University, Cambridge, UK ${ }^{2}$ Hinchingbrooke Hospital NHS Trust, Huntingdon, Cambridgeshire, UK

\section{Correspondence to} Professor Rupert R A Bourne, Vision \& Eye Research Unit, East Road, Anglia Ruskin University, Cambridge CB1 1PT, UK; rb@rupertbourne.co.uk

Received 15 March 2016 Revised 6 October 2016 Accepted 8 November 2016 Published Online First 7 December 2016

\begin{abstract}
Aims To evaluate temporal change in anterior chamber angle anatomy following argon laser peripheral iridoplasty (ALPI) in eyes with occludable angles postlaser peripheral iridotomy (LPI) compared with control eyes. Additionally, the effect on diurnal intraocular pressure (DIOP) fluctuation (maximumminimum (OP) was investigated.

Methods Twenty-two patients with bilateral primary angle closure/suspects with gonioscopically occludable anterior chamber angles following LPI were randomised to receive $A L P I(n=11)$ or no further treatment $(n=11)$. Angle opening distance (AOD), trabecular-iris angle, angle recess area and trabecular-iris space area were measured over eight sections with swept-source anterior segment optical coherence tomography and DIOP was measured pre-LPI and repeated at 3 months after ALPI (hourly measures).
\end{abstract}

Results All angle parameters increased following ALPI. This change was maintained for 3 months in seven of the eight sections (eg, inferotemporal AOD500 increased by $0.063 \mathrm{~mm}, p=0.004$ at 1 day; $0.051 \mathrm{~mm}, \mathrm{p}=0.029$ at 1 week; $0.059 \mathrm{~mm}, \mathrm{p}=0.006$ at 6 weeks and $0.056 \mathrm{~mm}, \mathrm{p}=0.011$ at 3 months). The only exception was in the inferior sector (eg, AOD500 increased by $0.041 \mathrm{~mm}, p=0.025$ at 1 day and by $0.029 \mathrm{~mm}$, $\mathrm{p}=0.054$ at 3 months). DIOP at 3 months was significantly reduced $(5.04 \mathrm{~mm} \mathrm{Hg} ; \pm 1.61 \mathrm{~mm} \mathrm{Hg}$ ) compared with controls $(6.61 \mathrm{~mm} \mathrm{Hg} ; \pm 1.63 \mathrm{~mm} \mathrm{Hg}$ ). Maximum IOP was significantly greater in the non-ALP group (1.87 mm Hg, $\mathrm{p}=0.026)$.

Conclusions ALPI widened all angle sections in eyes that remained occludable post-LPI. Changes were maintained for 3 months. ALPI decreased DIOP

fluctuation in the treated eyes by lowering the maximum IOP value.

\section{INTRODUCTION}

Argon laser peripheral iridoplasty (ALPI) has been used in the treatment of both acute primary angle closure (PAC) and angle closure glaucoma (PACG). ${ }^{1-4}$ ALPI involves placing a ring of laser burns on the peripheral iris to contract the iris stroma near the angle and has been found to be effective in widening the anterior chamber angles of eyes presenting with plateau iris syndrome, where the angle remains occludable after laser peripheral iridotomy (LPI). ${ }^{5}$ Leung et $a l^{6}$ described a PACG case in which the angle configuration changed from occludable to non-occludable following ALPI when the angle was measured with time- domain ocular coherence tomography. Recently, a widening of the angle at $750 \mu \mathrm{m}$ from the scleral spur in 15 non-Caucasian patients with acute PAC in a single horizontal cross-section was observed 1 hour post-ALPI as an only treatment. ${ }^{7}$

When treating acute angle closure cases, ALPI has shown to be more effective in lowering intraocular pressure (IOP) than systemic medications immediately after an angle closure attack. ${ }^{3}$ The IOP control following ALPI was maintained over a 3-month period. ${ }^{8}$ The mechanism of lowering of IOP by ALPI is likely to be mainly due to reduction of the area of trabecular meshwork that is occluded by the iris. The effect of ALPI on diurnal intraocular pressure (DIOP) fluctuation has not been investigated. We have shown previously that there is an inverse association between DIOP fluctuation and the dimensions of the anterior chamber angle in untreated patients with a diagnosis of primary angle closure suspect (PACS) or PAC in both eyes. Considering the use of ALPI in the clinical management of patients with occludable angles, it is important to understand if ALPI affects DIOP fluctuation in addition to the dimensions of the anterior chamber angle.

The purpose of this study was to evaluate temporal change in anterior chamber angle anatomy following ALPI in eyes with gonioscopically occludable angles post-LPI compared with eyes receiving no further treatment, using swept-source anterior segment optical coherence tomography (SS-OCT). Additionally, the effect of ALPI treatment on DIOP fluctuation (maximum-minimum IOP) was also investigated.

\section{METHODS}

This was a longitudinal, prospective, doublerandomised research study as part of the Investigating Management of Primary Angle Closure and Treatment (IMPACT) study. The aim of the IMPACT study is to identify factors that affect risk of conversion between PACS, PAC and PACG as well as to understand the effects of laser iridotomy and argon laser iridoplasty on the dimensions of anterior chamber in those eyes. IOP and the presence or absence of peripheral anterior synechiae (PAS) are used to categorise a patient into differing diagnostic categories. These categories reflect a differing risk for glaucoma-categorised individuals such as PACS, where only an occludable angle is present, and PAC, in which PAS and/or a raised IOP are additionally observed. 
Forty Caucasian consecutive patients newly referred to a hospital glaucoma service with a gonioscopic diagnosis $\left(<180^{\circ}\right.$ posterior pigmented trabecular meshwork visible on applanation gonioscopy) of bilateral PAC, PACS or a combination of both conditions and no other ocular comorbidity were recruited to the IMPACT study.

LPI procedures were performed by a single operator (RB) using a standard technique with superior placement of the iridotomy in a randomly allocated eye of each patient. The randomisation allowed the untreated eye to be used as control and patients were closely monitored for the duration of the study to monitor for signs of progression. The mean total power used to perform the iridotomy was $16.11 \mathrm{~mJ}$ (SD $10.8 \mathrm{~mJ}$ ) and the mean number of shots was 13 (SD $8.6 \mathrm{~mJ}$ ). A patent iridotomy post-LPI was present in all the treated eyes post-LPI and throughout the study. Patency was tested at the slit-lamp using a retroillumination technique.

The second randomisation (where eyes with gonioscopically closed anterior chamber angles were randomised to ALPI or no further treatment) took place 3 months post-LPI. A gonioscopically occludable anterior chamber angle was defined as an angle in which $180^{\circ}$ or more of the posterior trabecular meshwork was obscured on applanation gonioscopy. Gonioscopy examination was performed by a single examiner (RB) using standardised protocol. Patients were followed after ALPI treatment for 3 months with regular SS-OCT measurements and single IOP measurements until the final visit for DIOP measurements. A gonioscopically occludable anterior chamber angle was defined as an angle in which $180^{\circ}$ or more of the posterior trabecular meshwork was obscured on applanation gonioscopy. An overview of the patient pathway is given in figure 1 .

Prior to ALPI, one drop of topical pilocarpine (2\%) was instilled into the eye $15 \mathrm{~min}$ before the laser procedure which was performed (all by the same surgeon, RB) using an Abraham lens (Ocular Instruments, Bellevue, Washington, USA) under topical anaesthesia. Twenty laser spots were applied using the following laser settings: $0.5 \mathrm{~s}$ duration, $500 \mu$ spot size and 180-300 mW power.
Three-dimensional SS-OCT (Casia SS-1000 optical coherence tomograph; Tomey, Nagoya, Japan) images were obtained on the same day as the IOP measurements. A three-dimensional image of the anterior segment of the patient's eye was obtained by high-speed scanning without making a contact with the eye. The infrared measurement laser emitted to the patient's eye resulted in the acquired tomographic image of the eye displayed on the OCT monitor. After measurements were completed, the data were automatically transferred to the computer.

The scans were taken in darkness (between 0.3 and 0.5 lux) and the images taken were subsequently analysed using the commercially available software with this instrument. Image acquisition was always by the same examiner and an ophthalmologist subspecialising in glaucoma performed all the gonioscopic examinations (RB). The scleral spur position was identified manually by two independent observers with good concordance. The observers were not masked to the intervention and in case of disagreement, a third observer was asked to review the position of the scleral spur.

The analysis of SS-OCT images involved calculation of the following parameters in each eye: the angle opening distance (AOD), the trabecular-iris angle (TIA), the angle recess area (ARA) and the trabecular-iris space area (TISA). Eight sectors (superior, superonasal, nasal, inferonasal, inferior, inferotemporal, temporal and superotemporal) for every eye with their corresponding eight parameters (AOD, ARA, TISA and TIA, each at 500 and $750 \mu \mathrm{m}$ ) were assessed with the Casia analysis software (figure 2).

Following recruitment to the study, participants attended for IOP measurement every hour from 9:00 to 16:00 (a time window of $15 \mathrm{~min}$ around each clock hour was permitted). IOP measurements involved Goldmann tonometry (Goldman tonometer AT900; Haag-Streit International, Koeniz, Switzerland) using disposable prisms to reduce the risk of crosscontamination. The same tonometer was used for every IOP measurement for every participant and regular calibration checks were undertaken, with no calibration errors detected during the study. Two IOP measurements were taken per eye,

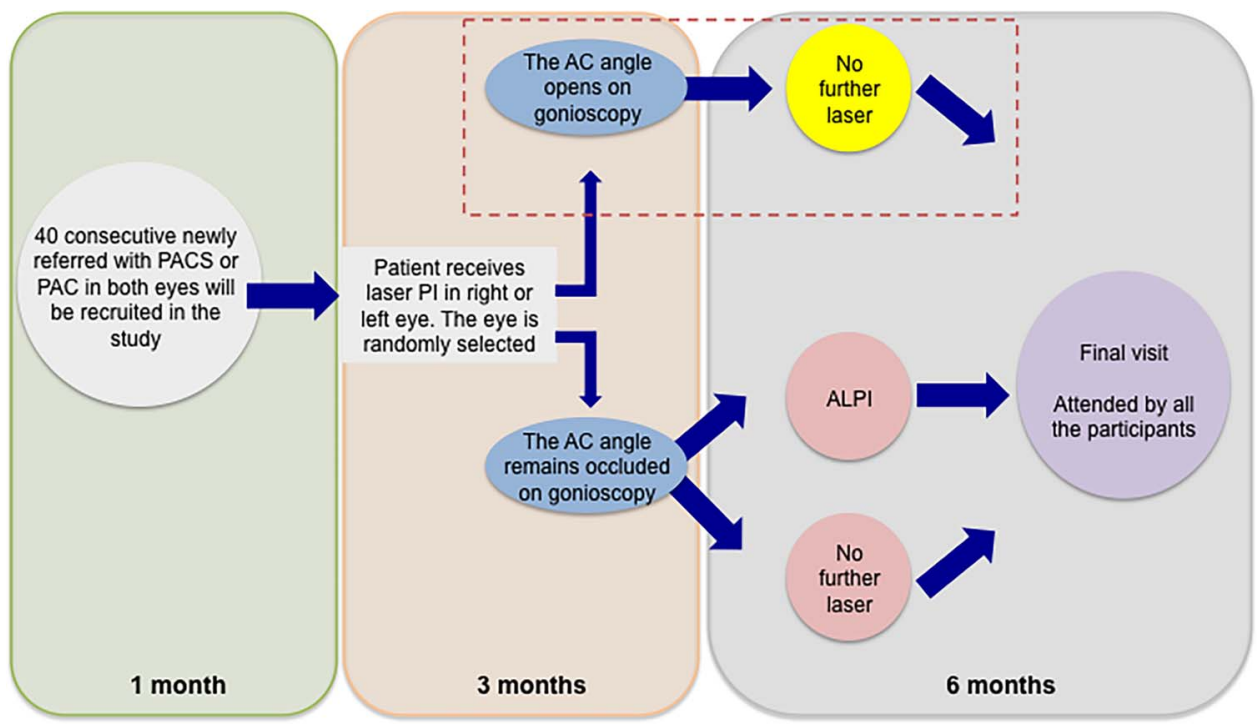

Figure 1 Schematic of IMPACT study pathway. AC, Anterior Chamber, PAC, primary angle closure, PACS, primary angle closure suspects, LPI, laser peripheral iridotomy, ALPI, argon laser peripheral iridoplasty. Patients with angles no longer judged as occludable on gonioscopy following LPI (red outline) were excluded from further analysis of effect of ALPI from time of treatment. 


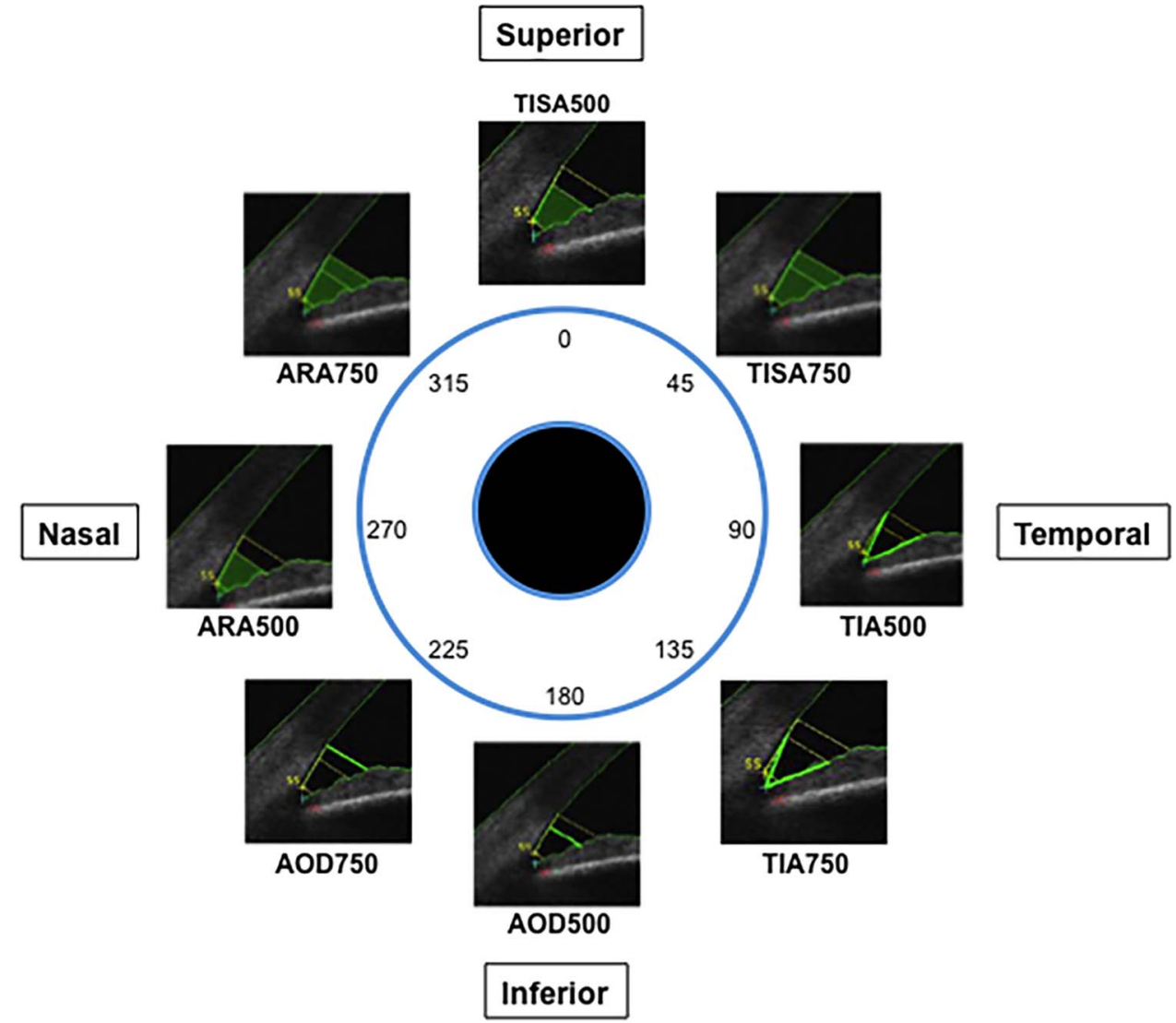

Figure 2 Schematic representation of the eight iridotrabecular angle sections under study. Iridotrabecular angle parameters as measured with the Casia AS-OCT analysis software. AOD, angle opening distance, ARA, angle recess area, TISA, trabecular-iris space area, TIA, trabecular-iris angle at 500 and $750 \mu \mathrm{m}$ are highlighted in bright green.

with a maximum of $1 \mathrm{~mm} \mathrm{Hg}$ difference permitted between these measurements.

Statistical analyses were performed using SPSS software Version 20 (IBM, Armonk, New York, USA) and Microsoft Office Excel software (Microsoft, Redmond, Washington, USA) with $\mathrm{p}<0.05$ values considered statistically significant. Angle width-related measures at different visits before and after LPI were compared using one-way repeated-measures analysis of variance, with intervisit difference analysed using Tukey's method.

Ethical approval by Cambridgeshire Research Ethics Committee (REC) for the IMPACT study was obtained on 3 August 2010 (REC Reference 10/H0301/14). The study was entered on the National Institute for Health Research Clinical Research Network (NIHR CRN) Portfolio on 9 September 2010 (NIHR CRN Study ID: 8955). The research adhered to the tenets of the Declaration of Helsinki.

\section{RESULTS}

The anterior chamber angles of LPI-treated eyes of 22 patients remained gonioscopically occludable. These eyes were randomly assigned to receive ALPI treatment $(n=11)$ or no further treatment $(n=11)$. We observed a statistically widening effect in 1 week in all parameters and sections following ALPI treatment (measurements obtained for AOD are presented in table 1; measurements obtained for ARA are presented in table 2; for additional angle parameters TISA and TIA, see online supplementary tables S1 and S2, respectively).
Table 1 Parameters from swept-source optical coherence tomography-measured anterior chamber angle sections before and after argon laser peripheral iridoplasty (ALPI)

\begin{tabular}{|c|c|c|c|}
\hline & Pre-ALPI* & $\begin{array}{l}1 \text { day } \\
\text { post-ALPIt }\end{array}$ & $\begin{array}{l}3 \text { months } \\
\text { post-ALPIt }\end{array}$ \\
\hline Superior AOD500 & 0.051 & $0.105(0.003)$ & $0.083(0.015)$ \\
\hline Superionasal AOD500 & 0.057 & $0.120(0.001)$ & $0.088(0.044)$ \\
\hline Nasal AOD500 & 0.135 & $0.179(0.002)$ & $0.160(0.012)$ \\
\hline Inferonasal AOD500 & 0.094 & $0.184(0.005)$ & $0.148(0.001)$ \\
\hline Inferior AOD500 & 0.059 & $0.101(0.025)$ & $0.088(0.054)$ \\
\hline $\begin{array}{l}\text { Inferotemporal } \\
\text { AOD500 }\end{array}$ & 0.094 & $0.157(0.004)$ & $0.150(0.011)$ \\
\hline Temporal AOD500 & 0.099 & $0.149(0.003)$ & $0.161(0.009)$ \\
\hline $\begin{array}{l}\text { Superotemporal } \\
\text { AOD500 }\end{array}$ & 0.065 & $0.103(0.031)$ & $0.096(0.006)$ \\
\hline Superior AOD750 & 0.105 & $0.118(0.062)$ & $0.167(0.011)$ \\
\hline Superionasal AOD750 & 0.093 & $0.145(0.004)$ & $0.123(0.046)$ \\
\hline Nasal AOD750 & 0.191 & $0.212(0.059)$ & $0.264(0.025)$ \\
\hline Inferonasal AOD750 & 0.189 & $0.257(0.007)$ & $0.199(0.05)$ \\
\hline Inferior AOD750 & 0.108 & $0.208(0.022)$ & $0.142(0.063)$ \\
\hline $\begin{array}{l}\text { Inferotemporal } \\
\text { AOD750 }\end{array}$ & 0.187 & $0.203(0.036)$ & $0.234(0.021)$ \\
\hline Temporal AOD750 & 0.149 & $0.209(0.004)$ & $0.183(0.044)$ \\
\hline $\begin{array}{l}\text { Superotemporal } \\
\text { AOD750 }\end{array}$ & 0.050 & $0.108(0.006)$ & $0.119(0.003)$ \\
\hline
\end{tabular}

${ }^{*}$ Mean measurements are shown.

tMean measurements at the indicated time point and paired t-test $p$ values of significance (in brackets) when comparing mean angle measurement at the indicated time point with the pre-ALPI measurement. AOD, angle opening distance. 
Table 2 Parameters from swept-source OCT-measured anterior chamber angle sections before and after argon laser peripheral iridoplasty (ALPI)

\begin{tabular}{|c|c|c|c|}
\hline & Pre-ALPI* & $\begin{array}{l}1 \text { day } \\
\text { post-ALPIt }\end{array}$ & $\begin{array}{l}3 \text { months } \\
\text { post-ALPIt }\end{array}$ \\
\hline Superior ARA500 & 0.018 & $0.046(0.020)$ & $0.036(0.013)$ \\
\hline Superonasal ARA500 & 0.033 & $0.063(0.044)$ & $0.051(0.049)$ \\
\hline Nasal ARA500 & 0.072 & $0.108(0.012)$ & $0.89(0.017)$ \\
\hline Inferonasal ARA500 & 0.052 & $0.088(0.019)$ & $0.072(0.045)$ \\
\hline Inferior ARA500 & 0.023 & $0.069(0.029)$ & $0.042(0.049)$ \\
\hline $\begin{array}{l}\text { Inferotemporal } \\
\text { ARA500 }\end{array}$ & 0.059 & $0.097(0.016)$ & $0.089(0.006)$ \\
\hline Temporal ARA500 & 0.055 & $0.091(0.035)$ & $0.085(0.019)$ \\
\hline $\begin{array}{l}\text { Superotemporal } \\
\text { ARA500 }\end{array}$ & 0.028 & $0.055(0.025)$ & $0.036(0.068)$ \\
\hline Superior ARA750 & 0.038 & $0.087(0.020)$ & $0.69(0.044)$ \\
\hline Superionasal ARA750 & 0.053 & $0.113(0.022)$ & $0.079(0.035)$ \\
\hline Nasal ARA750 & 0.116 & $0.180(0.065)$ & $0.163(0.040)$ \\
\hline Inferonasal ARA750 & 0.091 & $0.163(0.016)$ & $0.128(0.051)$ \\
\hline Inferior ARA750 & 0.041 & $0.133(0.021)$ & $0.051(0.021)$ \\
\hline $\begin{array}{l}\text { Inferotemporal } \\
\text { ARA750 }\end{array}$ & 0.101 & $0.168(0.044)$ & $0.143(0.007)$ \\
\hline Temporal ARA750 & 0.088 & $0.150(0.002)$ & $0.127(0.013)$ \\
\hline $\begin{array}{l}\text { Superotemporal } \\
\text { ARA750 }\end{array}$ & 0.050 & $0.101(0.043)$ & $0.089(0.003)$ \\
\hline
\end{tabular}

${ }^{*}$ Mean measurements are shown.

tMean measurements at the indicated time point and paired $t$-test $p$ values of significance (in brackets) when comparing mean angle measurement at the indicated time point with the pre-ALPI measurement. ARA, angle recess area.

For example, at 1 day following ALPI treatment AOD500 increased in the superior sector by $0.054 \mathrm{~mm}, \mathrm{p}=0.003$; superonasal sector by $0.062 \mathrm{~mm}, \mathrm{p}=0.001$; nasal sector by $0.044 \mathrm{~mm}$, $\mathrm{p}=0.002$; inferonasal sector by $0.082 \mathrm{~mm}, \mathrm{p}=0.005$; inferior sector by $0.041 \mathrm{~mm}, \mathrm{p}=0.025$; inferotemporal sector by $0.063 \mathrm{~mm}, \mathrm{p}=0.004$; temporal sector by $0.051 \mathrm{~mm}, \mathrm{p}=0.003$ and superotemporal sector by $0.038 \mathrm{~mm}, \mathrm{p}=0.031$. This increase in AOD500 dimensions was maintained over time for 3 months (eg, inferotemporal segment AOD500 increased by $0.063 \mathrm{~mm}, \mathrm{p}=0.004$ at 1 day; $0.051 \mathrm{~mm}, \mathrm{p}=0.029$ at 1 week; $0.059 \mathrm{~mm}, \mathrm{p}=0.006$ at 6 weeks and $0.056 \mathrm{~mm}, \mathrm{p}=0.011$ at
12 weeks). The only exception was observed in the inferior sector, where there was no statistically significant difference at 3 months $(0.041 \mathrm{~mm}, \mathrm{p}=0.025$ at 1 day and $0.029 \mathrm{~mm}$, $\mathrm{p}=0.054$ at 3 months) (figure 3 ).

Similar results were observed for ARA500, TISA500 and TIA500 (see online supplementary materials). The widening of angle parameters at $750 \mu \mathrm{m}$ from the scleral spur was similar to that observed for all parameters at $500 \mu \mathrm{m}$ from scleral spur (figure 4). A schematic showing temporal changes in angle parameters as visualised by SS-OCT is given in figure 5, showing the angle prior to ALPI treatment, 1 month after ALPI treatment and 3 months after ALPI treatment.

DIOP fluctuation prior to ALPI was significantly greater than 3 months post-ALPI (mean difference, $1.56 \mathrm{~mm} \mathrm{Hg}, \mathrm{p}=0.047$ ). Further analyses were undertaken in order to determine if this difference in DIOP fluctuation was due to a difference between diurnal maxima, diurnal minima or both. In a comparison of ALPI-treated and ALPI-untreated eyes that had previous iridotomy, the maximal values (treated maximum $\mathrm{IOP}=19.34 \mathrm{~mm} \mathrm{Hg}, \quad \pm 1.58 \mathrm{~mm} \mathrm{Hg}$ at 12 weeks; untreated maximum $\mathrm{IOP}=21.15 \mathrm{~mm} \mathrm{Hg}, \pm 1.52 \mathrm{~mm} \mathrm{Hg}$ ) and the comparison of the minimum values (treated minimum $\mathrm{IOP}=14.23 \mathrm{~mm} \mathrm{Hg}, \quad \pm 2.00 \mathrm{~mm} \mathrm{Hg}$; untreated minimum $\mathrm{IOP}=14.54 \mathrm{~mm} \mathrm{Hg}, \pm 1.88 \mathrm{~mm} \mathrm{Hg}$ ) confirmed that the difference in DIOP fluctuation was due to maximum values rather than minimum values (figure 6).

Analysis of covariance comparing ALPI-treated eyes with the fellow eyes, which received no laser treatment in the study, showed no statistically significant differences in comparison to the DIOP minima between groups (mean difference between minima DIOP $=0.31 \mathrm{~mm} \mathrm{Hg} ; \mathrm{p}=0.058$ ). However, in a comparison of the DIOP maxima, the mean difference between groups was found to be statistically significant of $1.87 \mathrm{~mm} \mathrm{Hg}$, $\mathrm{p}=0.026$ ) with the untreated eyes exhibiting higher average maximum IOP measurements.

\section{DISCUSSION}

All angle parameters under study increased following LPI as measured by SS-OCT prior to the ALPI randomisation (data submitted elsewhere for publication). In the eyes that underwent ALPI due to the angle remaining gonioscopically occludable, following the ALPI treatment all eyes changed from an
Figure 3 Changes in angle opening distance at $500 \mu \mathrm{m}$ from scleral spur in all eight sections of the anterior chamber angle (11 eyes) in argon laser peripheral iridoplasty-treated eyes measured with swept-source optical coherence tomography in dark conditions.

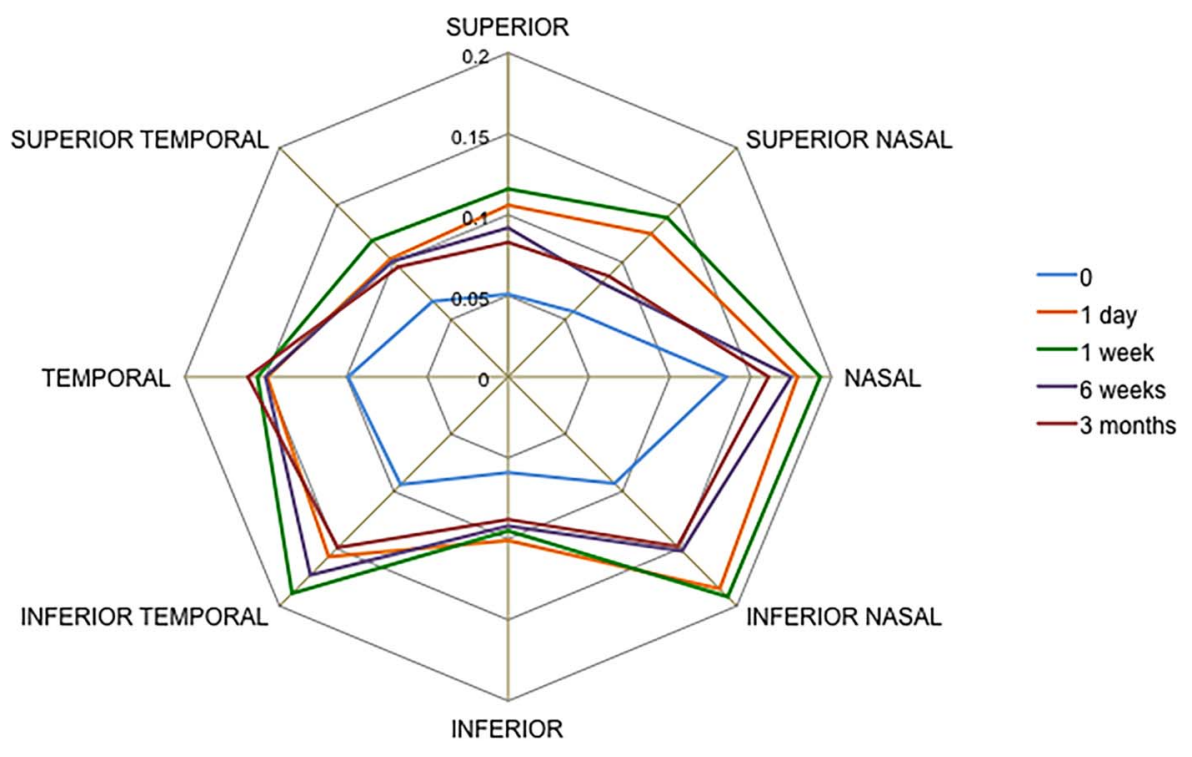


Figure 4 Changes in angle opening distance at $750 \mu \mathrm{m}$ from scleral spur in all eight sections of the anterior chamber angle (11 eyes) in argon laser peripheral iridoplasty-treated eyes measured with swept-source optical coherence tomography in dark conditions.

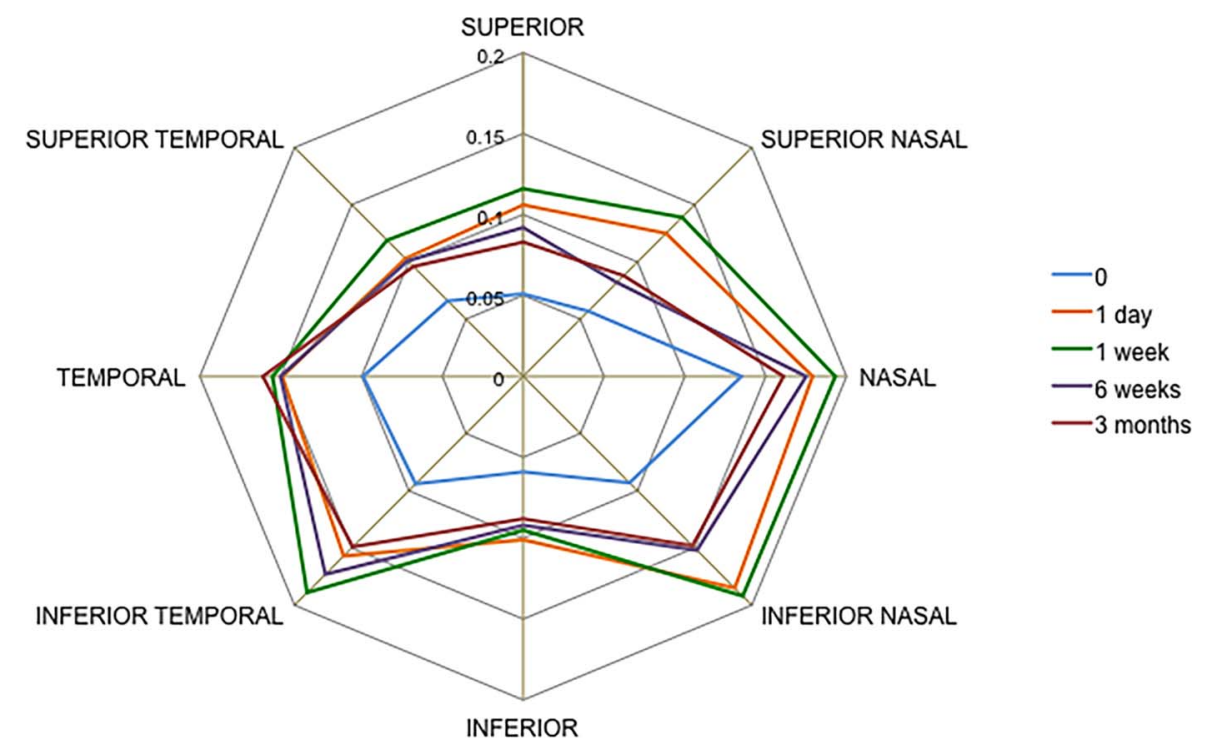

Chew and Yeo $^{1}$ investigated the IOP levels of 11 ALPI-treated PACG participants whose angles remained occludable following LPI. They observed an initial reduction of IOP during the first week post-treatment, but a final comparison with baseline at 6 months following treatment showed a maintained decrease in IOP in only seven of the treated eyes. However, it is difficult to compare these results with our data as the time of day for the IOP assessment was not specified, their patients had PACG and it was not evident that the timing of the IOP measurement was kept constant from visit to visit. Attention to this detail has been demonstrated to reduce the intervisit variability in IOP measures. ${ }^{10}$ Similar limitations may apply in a recent study by Sun et $a l,{ }^{11}$ where 158 PAC/PACG participants were randomised into two groups as follows: (1) LPI alone or (2) LPI followed by ALPI. The IOP in the study was measured at baseline and at seven more visits during the following year. The authors report a reduction in absolute IOP of approximately $6.7 \mathrm{~mm} \mathrm{Hg}$ in the LPI-treated group and $7.8 \mathrm{mmHg}$ in the LPI plus ALPI group 12 months following the laser treatment. However, it is not clear if these IOP measurements were taken at the same time of the day as in the prelaser visit and therefore whether the observed reduction in IOP following the laser procedures would fall within the limits of the patient's usual diurnal IOP fluctuation. Lee $e t a l^{12}$ randomised one eye to receive LPI and the fellow eye to receive LPI plus ALPI (same setting) in patients with bilateral PACS and reported that the anterior chamber depth measured at 4-6 $\mathrm{mm}$ from the centre of the eye was significantly different between the two treatment groups. The measurements were performed 1 week after the procedure and assessed with Pentacam. No statistically significant differences in
Figure 5 Temporal changes in angle parameters as visualised by SS-OCT.

(A) Prior to argon laser peripheral iridoplasty (ALPI) treatment, (B)

1 month after ALPI treatment, (C)

3 months after ALPI treatment.
A

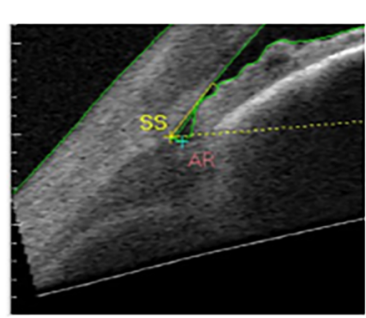

Prior to ALPI

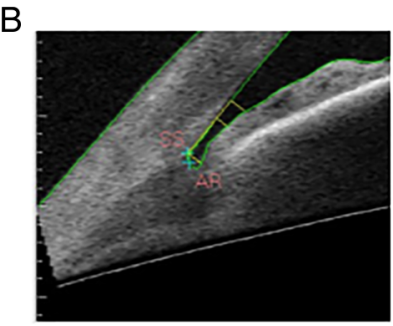

1 month post-ALPI

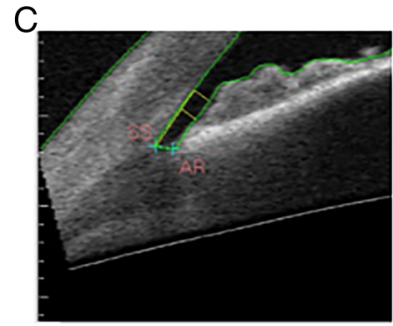

3 months post-ALPI 


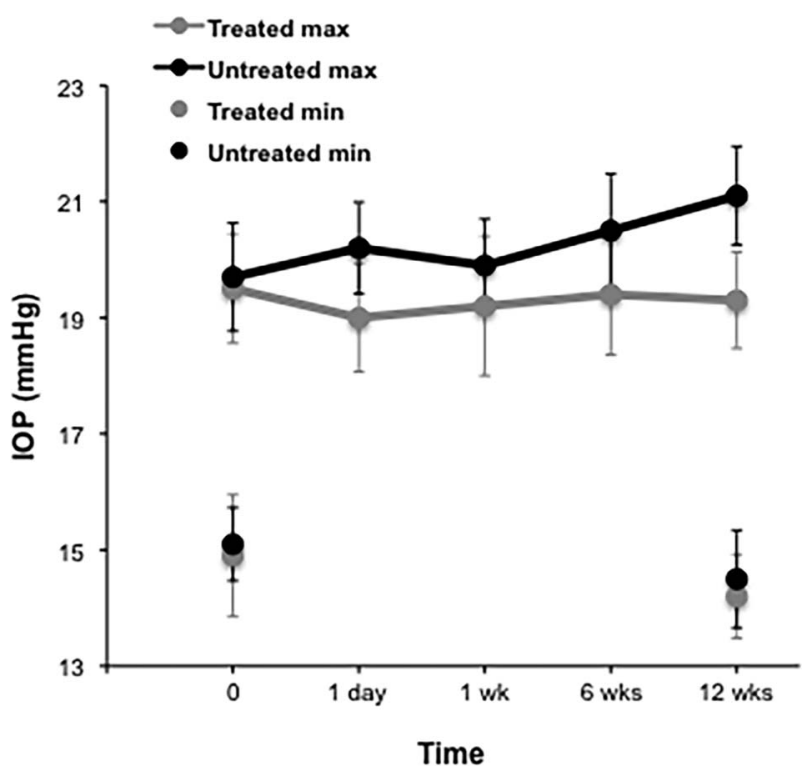

Figure 6 Intraocular pressure (IOP) measurements in treated (argon laser peripheral iridoplasty) and untreated eyes over 6 months, displayed as diurnal IOP (maximum-minimum IOP) and maximal and minimal IOP separately, y error bars indicate SE.

IOP measurements were found between baseline and 1 hour, 1 day, 1 week, 1 month and 3 months post-treatment. However, the time of IOP measurement again was not specified. A recent study by Narayanaswamy et $a l^{13}$ reported that in eyes with persistent appositional angle closure and raised IOP, ALPI reduced IOP on average by $4.9 \mathrm{~mm} \mathrm{Hg}$ and in $35 \%$ of the patient achieved IOP of $<21 \mathrm{~mm} \mathrm{Hg}$ without medication. However, when assessed at 1 year following treatment, ALPI was associated with higher failure rates and lower IOP reduction compared with medical treatment. The comparison of their results with the changes we observe in the angle parameters will be difficult due to the shorter follow-up time and smaller number of patients in our study.

In this study, we have demonstrated that ALPI is effective in lowering DIOP fluctuation by $1.56 \mathrm{~mm} \mathrm{Hg} \quad(p=0.047)$ at 12 weeks post-treatment compared with before treatment. The difference in DIOP fluctuation was due to the lower maximum measurements in the ALPI group $(1.87 \mathrm{~mm} \mathrm{Hg}, \mathrm{p}=0.026)$ compared with the occludable post-LPI group. Furthermore, in our study the ALPI-treated group of eyes showed the lowest DIOP fluctuation compared with the non-treated control eyes, eyes deemed not occludable post-LPI and eyes that were occludable post-LPI, demonstrating that ALPI significantly reduces DIOP fluctuation.

\section{CONCLUSION}

ALPI increased all angle parameters under investigation in all eight sections in eyes that remained occludable post-LPI. The changes in angle dimension detected using SS-OCT were also confirmed on gonioscopy. The increase in angle parameters was maintained for 12 weeks with the exception of the inferior segment where some regression in the evaluated parameters was observed at 12 weeks. The finding that ALPI significantly widens the anterior chamber angle circumferentially may confer protection from the onset or progression of existing damage to the trabecular meshwork. The reduction in DIOP fluctuation caused by the ALPI is likely to be related to these changes in anterior chamber dimensions.

Acknowledgements We wish to acknowledge the work of Laura Sanchez Parra in data collection and Paula Turnbull and Heather Pearman for administration of the study. Additionally, Professor Roger Buckley gave advice on aspects of the study design and Michael Parker provided statistical advice. Tomey Corporation (Nagoya, Japan) loaned the instrument for the purposes of the study.

Contributors RRAB was involved in the conception and design of the study. RRAB contributed to the acquisition, analysis and interpretation of data as well as drafting the work and revising it critically. RRAB has provided final approval of the version to be published. IZ contributed to the acquisition, analysis and interpretation of data as well as drafting the work and revising it critically. IZ has provided final approval of the version to be published.

SP was involved in the conception and design of the study. SP contributed to the analysis and interpretation of data as well as revising the work critically. SP has provided final approval of the version to be published.

Funding This research was supported by Hinchingbrooke Hospital Ophthalmology Research Fund.

Competing interests None declared.

Patient consent Obtained.

Ethics approval Research Ethics Committee.

Provenance and peer review Not commissioned; externally peer reviewed.

Open Access This is an Open Access article distributed in accordance with the Creative Commons Attribution Non Commercial (CC BY-NC 4.0) license, which permits others to distribute, remix, adapt, build upon this work non-commercially, and license their derivative works on different terms, provided the original work is properly cited and the use is non-commercial. See: http://creativecommons.org/ licenses/by-nc/4.0/

\section{REFERENCES}

1 Chew PT, Yeo LM. Argon laser iridoplasty in chronic angle closure glaucoma. Int Ophthalmol 1995;19:67-70.

2 Lai JS, Tham CC, Chua JK, et al. Immediate diode laser peripheral iridoplasty as treatment of acute attack of primary angle closure glaucoma: a preliminary study. J Glaucoma 2001;10:89-94.

3 Lam DS, Lai JS, Tham CC, et al. Argon laser peripheral iridoplasty versus conventional systemic medical therapy in treatment of acute primary angle-closure glaucoma: a prospective, randomized, controlled trial. Ophthalmology 2002;109:1591-6.

4 Lee JW, Lai JS, Yick DW, et al. Argon laser peripheral iridoplasty versus systemic intraocular pressure-lowering medications as immediate management for acute phacomorphic angle closure. Clin Ophthalmol 2013;7:63-9.

5 Ritch R, Tham CC, Lam DS. Argon laser peripheral iridoplasty (ALPI): an update. Surv Ophthalmol 2007:52:279-88.

6 Leung CK, Chan WM, Ko CY, et al. Visualization of anterior chamber angle dynamics using optical coherence tomography. Ophthalmology 2005;112:980-4.

7 Sng CC, Aquino MC, Liao J, et al. Anterior segment morphology after acute primary angle closure treatment: a randomised study comparing iridoplasty and medical therapy. Br J Ophthalmol 2016;100:542-8.

8 Lai JS, Tham CC, Chua JK, et al. To compare argon laser peripheral iridoplasty (ALPI) against systemic medications in treatment of acute primary angle-closure: mid-term results. Eye (Lond) 2006;20:309-14.

9 Sanchez-Parra L, Pardhan S, Buckley RJ, et al. Diurnal intraocular pressure and the relationship with swept-source OCT-derived anterior chamber dimensions in angle closure: the IMPACT study. Invest Ophthalmol Vis Sci 2015;56:2943-9.

10 Rotchford AP, Uppal S, Lakshmanan A, et al. Day-to-day variability in intraocular pressure in glaucoma and ocular hypertension. Br J Ophthalmol 2012;96:967-70.

11 Sun $X$, Liang YB, Wang NL, et al. Laser peripheral iridotomy with and without iridoplasty for primary angle-closure glaucoma: 1-year results of a randomized pilot study. Am J Ophthalmol 2010;150:68-73.

12 Lee JR, Choi JY, Kim YD, et al. Laser peripheral iridotomy with iridoplasty in primary angle closure suspect: anterior chamber analysis by pentacam. Korean J Ophthalmol 2011;25:252-6.

13 Narayanaswamy A, Baskaran M, Perera SA, et al. Argon laser peripheral iridoplasty for primary angle-closure glaucoma: a randomised control trial. Ophthalmology 2016;123: 514-21. 